\title{
Room-Temperature Zwitterionic Ionic Liquids
}

\author{
Romain Bordes, ${ }^{a}$ Jean-Daniel Marty ${ }^{a}$ and Nancy Lauth-de Viguerie ${ }^{a^{*}}$ \\ ${ }^{a}$ Laboratoire des IMRCP, University of Toulouse, CNRS UMR 5623, 118 route de Narbonne, 31062 \\ Toulouse cedex 9, France \\ viguerie@chimie.ups-tlse.fr
}

Keywords: zwitterionic ionic liquids, urocanic acid, (E)-3-(1H-imidazol-4-yl)-2-propionic acid, photoisomerization, imidazolium

A novel series of zwitterionic ionic liquids based on $(E)$ or $(Z)$ isomer of an urocanic moiety containing a carboxylate group have been prepared. All synthesized compounds present melting points below $100^{\circ} \mathrm{C}$. This value can be easily tuned by changing the length of alkyl chain grafted on the imidazolium moiety and the stereochemistry of isomer. Hence, melting temperature as low as $20^{\circ} \mathrm{C}$ was obtained for $Z$ isomer with a N1, N3-methyloctyl imidazolium moiety.

\section{Introduction}

Room Temperature Ionic Liquids (ILs) are tuneable and generally environmentally benign solvents that enable to tackle scientific challenges for the industrial implementation of green innovations [1]. Various IL structures were designed to adjust their physicochemical properties to specific needs in many applications [2,3]. Nevertheless, the presence of free ions can be an issue in some cases, e.g. for the study of nucleophilic reactions or in electrochemical applications where migration of IL component ions is prejudicial. Accordingly, a class of ionic liquids in which both cation and anion units tethered covalently, called Zwitterionic Ionic Liquids (ZILs) was developed. The most common zwitterionic-type ionic liquids are nitrogene heterocycles with sulfonate moiety whose examples of structures are given in Figure 1. These ZILs have been intensively studied as designable electrolyte materials for lithium batteries [4] and fuel cells [5].
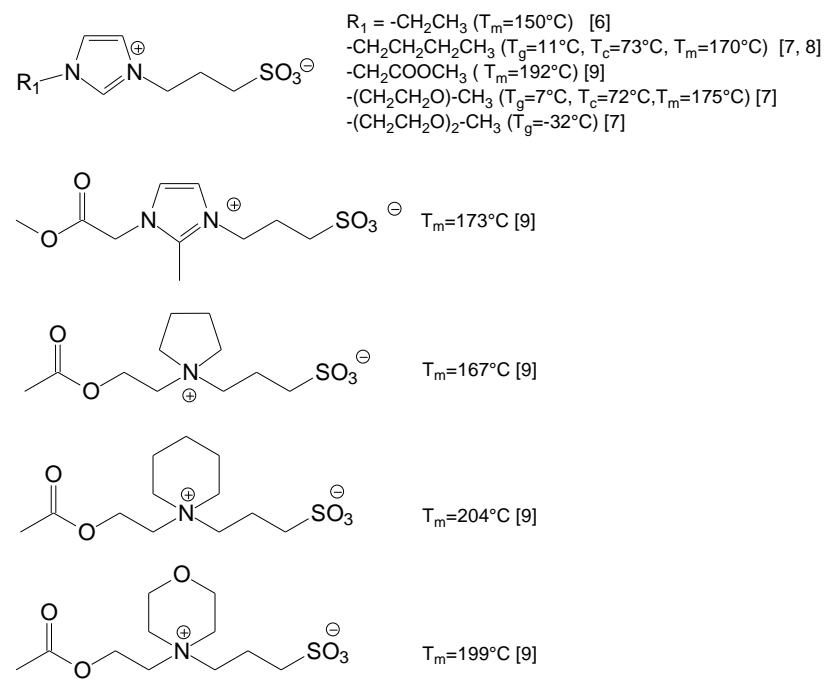

Figure 1. Some examples of zwitterionic-type molten salts containing a sulfonate group with corresponding melting temperature $\left(T_{m}\right)$, glass transition temperature $\left(T_{g}\right)$, and crystallization temperature $\left(\mathrm{T}_{\mathrm{c}}\right)$. 
Less explored ZILs are structures based on imidazoliums with carboxylate functions (Figure 2). They have been used as Bronsted acidic catalysts [10], for desulfurization of fuels [11], for metal oxides solubilization [12] or as liquid crystals [13].

The melting points of ZILs are generally higher than the ones of classical ILs because of a decrease in the motional freedom of each ion and strong intermolecular interactions (Figures $1-2)$.

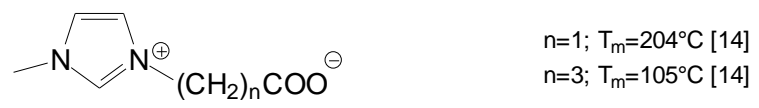

$\operatorname{HOOC}\left(\mathrm{CH}_{2}\right) \mathrm{n}^{-\mathrm{N}} \mathrm{N}_{\left(\mathrm{CH}_{2}\right)_{\mathrm{n}} \mathrm{COO}^{\ominus}} \begin{aligned} & \mathrm{n}=1 ; \mathrm{T}_{\mathrm{m}}=260^{\circ} \mathrm{C} \text { [14] } \\ & \mathrm{n}=3 ; \mathrm{T}_{\mathrm{m}=172^{\circ} \mathrm{C} \text { [14] }}\end{aligned}$<smiles>[R]n1cc[n+]([3H])c1C(=O)[O-]</smiles>

Figure 2. Some examples of zwitterionic-type molten salts containing a carboxylate group with corresponding melting temperature $\left(\mathrm{T}_{\mathrm{m}}\right)$.

All known examples of imidazolium salts bearing carboxylic acid groups have high melting points and therefore have had no application as reaction media.

We describe here the synthesis and characterization of a series of ionic liquids based on imidazolium moiety with a carboxylate function, with low melting points using a natural product as starting material $(E)$ 3-(1H-imidazol-4-yl)-2-propionic acid or (E)urocanic acid. This compound is a major metabolite of $L$-histidine found in the skin and excreted in sweat $[16,17]$. It is one of the major UV light absorbers in the epidermis where it acts as a natural photoprotecting agent [18]. Under irradiation, this compound undergoes photoisomerization to give a mixture of the $(E)$ and $(Z)$ isomers (Scheme 1). The $(Z)$ isomer has been found to have immunosuppressive activity. This compound presents an imidazole moiety which can be quaternized and a carboxylic function useful to prepare ZILs. Here, we describe the synthetic route for the preparation of ZILs based on urocanic moiety $E$ or $Z$.

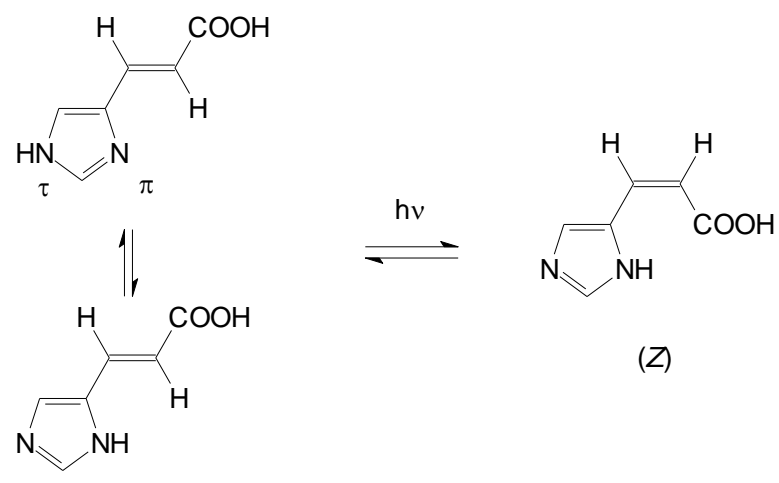

$(E)$

Scheme 1. Structure of the two tautomeric forms of $(E)$ urocanic acid and $E / Z$ photoisomerization

\section{Experimental part}

Material and methods.

All the solvents were purchased from Prolabo or Carlo Erba and were used without further purification. Reagents were purchased from Aldrich or Acros (>98\% purity). ${ }^{1} \mathrm{H}$ and ${ }^{13} \mathrm{C}$ RMN spectra were recorded on Brucker AC 250 and $\mathrm{AC} 400$ spectrometers. The $\mathrm{DCI} / \mathrm{NH}_{3}$ or $\mathrm{CH}_{4}$ mass spectra were recorded on a Nermag R10-10 apparatus and the FAB mass spectra on 
a ZAB-MS apparatus (WG-ANALYTICAL, Manchester UK). The thermal properties of the ZILs were determined by Differential Scanning Calorimetry (DSC) using a Perkin Elmer Pyris 1 calorimeter. Transition temperatures were taken at the onset of the DSC peaks as the temperature increase at different heating rates, 1, 2 and $5^{\circ} \mathrm{C} / \mathrm{min}$, and finally extrapolated to $0^{\circ} \mathrm{C} / \mathrm{min}$. The microanalyses were carried out at the LCC (Toulouse, France) on Perkin Elmer analyser 2400 series 2. Irradiation was carried out in a Rayonnet type RPP 100 apparatus equipped with a turntable.

Synthesis (see scheme 2 for notations)

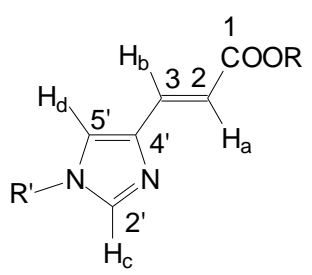

(E)-urocanic methyl ester. At room temperature, a suspension of $(E)$-urocanic acid $(7.5 \mathrm{~g}, 54.3$ mmol) in dry methanol $(250 \mathrm{~mL})$ was saturated with $\mathrm{HCl}$ gas. The solution obtained was refluxed through a Soxlet thimble containing activated $3 \AA$ sieves for $3 \mathrm{~h}$. The solvent was removed under reduced pressure giving the ester hydrochloride. The residue was neutralized by a saturated aqueous $\mathrm{NaHCO}_{3}$ solution, extracted with ethyl acetate $(3 \times 25 \mathrm{~mL})$ and dried over sodium sulfate. Evaporation of ethyl acetate gives $(E)$-urocanic methyl ester as a white solid in a $76 \%$ yield $(6.27 \mathrm{~g})$.
$R M N{ }^{l} H\left(\mathrm{CDCl}_{3}\right) \delta(\mathrm{ppm}): 3.72\left(\mathrm{~s}, \mathrm{OCH}_{3}, 3 \mathrm{H}\right)$; $6.44\left(\mathrm{AB}, J_{A B}=15.85 \mathrm{~Hz}, 1 \mathrm{H}, \mathrm{H}_{\mathrm{a}}\right) ; 7.20(\mathrm{~s}, 1 \mathrm{H}$, $\left.\mathrm{H}_{\mathrm{d}}\right) ; 7.55\left(\mathrm{AB}, J_{A B}=15.85 \mathrm{~Hz}, 1 \mathrm{H}, \mathrm{H}_{\mathrm{b}}\right) ; 7.65(\mathrm{~s}$, $\left.1 \mathrm{H}, \mathrm{H}_{\mathrm{c}}\right) \cdot R M N^{13} \mathrm{C}\left(\mathrm{CD}_{3} \mathrm{COCD}_{3}\right) \delta(\mathrm{ppm}): 50.56$ $\left(\mathrm{CH}_{3} \mathrm{O}\right) ; 114.26\left(\mathrm{C}_{2}\right) ; 122.10\left(\mathrm{C}_{5}{ }^{\prime}\right) ; 135.72\left(\mathrm{C}_{4}{ }^{\prime}\right)$; $136.11\left(\mathrm{C}_{3}\right) ; 137.36\left(\mathrm{C}_{2}\right) ; 167.21\left(\mathrm{C}_{1}\right) . M S(\mathrm{DCI}$, $\left.\mathrm{NH}_{3}\right): \mathrm{m} / \mathrm{z}=153, \mathrm{MH}^{+}(100 \%)$. Anal calc $(\%)$ for $\mathrm{C}_{7} \mathrm{H}_{8} \mathrm{~N}_{2} \mathrm{O}_{2}, 0.5 \mathrm{H}_{2} \mathrm{O}$ : C 52.17; $\mathrm{H} 5.62 ; \mathrm{N}$ 17.38; \% Found C 52.15; H 5.63; N 17.25. DSC: $\mathrm{T}_{\mathrm{m}}=92^{\circ} \mathrm{C}$.

$N \tau$-alkyl urocanic methyl ester $(\mathbf{1} \boldsymbol{a}, \mathbf{1 b}, \mathbf{1 c}, \mathbf{1 d})$

General procedure for $N \tau$-alkylation. At room temperature and under argon, $28 \mathrm{mmol}$ of 1bromoalkane were added dropwise to a mixture of $(E)$-urocanic methyl ester (14 mmol), $\mathrm{K}_{2} \mathrm{CO}_{3}$ (14 mmol) and 18-crown-6 (1.4 mmol) in 100 $\mathrm{mL}$ of anhydrous THF. The mixture was stirred $24 \mathrm{~h}$ at $60^{\circ} \mathrm{C}$. After filtration of the remaining $\mathrm{K}_{2} \mathrm{CO}_{3}$, THF was evaporated under reduced pressure. Purification by flash chromatography (silica gel, AcOEt/Et ${ }_{2} \mathrm{O} 50 / 50, \mathrm{~V} / \mathrm{V}$ ) afford to obtain $\mathrm{N} \tau$-alkyl urocanic methyl ester.

Compound 1a: Yield: $89 \%$. RMN ${ }^{1} \mathrm{H}\left(\mathrm{CDCl}_{3}\right)$ $\delta(\mathrm{ppm}): 0.95\left(\mathrm{t},{ }^{3} \mathrm{~J}=7.3 \mathrm{~Hz}, \mathrm{C}_{3} \underline{(}\left(\mathrm{CH}_{2}\right)_{3} \mathrm{~N}\right.$, $3 \mathrm{H}) ; 1.31\left(\mathrm{~m}, \mathrm{CH}_{3} \mathrm{CH}_{2}\left(\mathrm{CH}_{2}\right)_{2} \mathrm{~N}, 2 \mathrm{H}\right) ; 1.75(\mathrm{~m}$, $\left.\mathrm{CH}_{3} \mathrm{CH}_{2} \mathrm{CH}_{2} \mathrm{CH}_{2} \mathrm{~N}, 2 \mathrm{H}\right) ; 3.77\left(\mathrm{~s}, \mathrm{OCH}_{3}, 3 \mathrm{H}\right)$; $3.92\left(\mathrm{t},{ }^{3} \mathrm{~J}=7.3 \mathrm{~Hz}, \mathrm{CH}_{3}\left(\mathrm{CH}_{2}\right)_{2} \mathrm{C}_{2} \underline{\mathrm{N}}, 2 \mathrm{H}\right) ; 6.63$ $\left(\mathrm{AB}, J_{A B}=15.90 \mathrm{~Hz}, 1 \mathrm{H}, \mathrm{H}_{\mathrm{a}}\right) ; 7.26\left(\mathrm{~s}, 1 \mathrm{H}, \mathrm{H}_{\mathrm{d}}\right)$; $7.35\left(\mathrm{AB}, J_{A B}=15.90 \mathrm{~Hz}, 1 \mathrm{H}, \mathrm{H}_{\mathrm{b}}\right) ; 8.27(\mathrm{~s}, 1 \mathrm{H}$, $\left.\mathrm{H}_{\mathrm{c}}\right) . R M N{ }^{13} \mathrm{C}\left(\mathrm{CD}_{3} \mathrm{COCD}_{3}\right) \delta(\mathrm{ppm}): 13.41$ $\left(\mathrm{CH}_{3}\left(\mathrm{CH}_{2}\right)_{3} \mathrm{~N}\right) ; 1.63\left(\mathrm{CH}_{3} \underline{\mathrm{CH}_{2}}\left(\mathrm{CH}_{2}\right)_{2} \mathrm{~N}\right) ; 32.86$ 
$\left(\mathrm{CH}_{3} \mathrm{CH}_{2} \mathrm{CH}_{2} \mathrm{CH}_{2} \mathrm{~N}\right) ; 47.02\left(\mathrm{CH}_{3}\left(\mathrm{CH}_{2}\right)_{2} \underline{\mathrm{CH}_{2} \mathrm{~N}}\right)$; $51.39\left(\mathrm{CH}_{3} \mathrm{O}\right) ; 115.29\left(\mathrm{C}_{2}\right) ; 121.50 \quad\left(\mathrm{C}_{5}\right)$; $136,39\left(\mathrm{C}_{3}\right) ; 138,45\left(\mathrm{C}_{2}\right) ; 138,22\left(\mathrm{C}_{4}{ }^{\prime}\right) ; 168.08$ $\left(\mathrm{C}_{1}\right)$. MS : DCI $\left(\mathrm{NH}_{3}\right) \mathrm{m} / \mathrm{z}=209, \mathrm{MH}^{+}(100 \%)$. Anal calc (\%) for $\mathrm{C}_{11} \mathrm{H}_{16} \mathrm{~N}_{2} \mathrm{O}_{2}$ : C 63.44; $\mathrm{H}$ 7.74; N 13.45; \% Found C 64.35; H 7.73; N 13.43. DSC: $\mathrm{T}_{\mathrm{m}}=38^{\circ} \mathrm{C}$.

Compound 1b: Yield: $90 \% . R M N^{1} H\left(\mathrm{CDCl}_{3}\right) \delta$ (ppm): 0.86 (t, $\left.{ }^{3} \mathrm{~J}=6.7 \mathrm{~Hz}, \underline{\mathrm{C}}_{3}\left(\mathrm{CH}_{2}\right)_{5} \mathrm{~N}, 3 \mathrm{H}\right)$; $1.25\left(\mathrm{~m}, \mathrm{CH}_{3} \underline{\mathrm{CH}}_{2} \underline{\mathrm{CH}}_{2} \underline{\mathrm{CH}}_{2} \mathrm{C}_{2} \mathrm{CH}_{2} \mathrm{~N}, 8 \mathrm{H}\right) ; 1.75$ (m, $\left.\mathrm{CH}_{3}\left(\mathrm{CH}_{2}\right)_{3} \mathrm{CH}_{2} \mathrm{CH}_{2} \mathrm{~N}, 2 \mathrm{H}\right) ; 3,75\left(\mathrm{~s}, \mathrm{OCH}_{3}\right.$, $3 \mathrm{H}) ; 3.90\left(\mathrm{t},{ }^{3} \mathrm{~J}=7.3 \mathrm{~Hz}, \mathrm{CH}_{3}\left(\mathrm{CH}_{2}\right)_{4} \underline{\mathrm{C}}_{2} \mathrm{~N}\right.$, $2 \mathrm{H}) ; 6.52\left(\mathrm{AB}, J_{A B}=15,85 \mathrm{~Hz}, 1 \mathrm{H}, \mathrm{H}_{\mathrm{a}}\right) ; 707(\mathrm{~s}$, $\left.1 \mathrm{H}, \mathrm{H}_{\mathrm{d}}\right) ; 7.45\left(\mathrm{~s}, 1 \mathrm{H}, \mathrm{H}_{\mathrm{c}}\right) ; 7.53\left(\mathrm{AB}, J_{A B}=15,85\right.$ $\left.\mathrm{Hz}, 1 \mathrm{H}, \mathrm{H}_{\mathrm{b}}\right) . \mathrm{MS}: \mathrm{DCI}\left(\mathrm{NH}_{3}\right) \mathrm{m} / \mathrm{z}=237, \mathrm{MH}^{+}$ (100\%). Anal calc (\%) for $\mathrm{C}_{13} \mathrm{H}_{20} \mathrm{~N}_{2} \mathrm{O}_{2}$ : C $66.07 ; \mathrm{H} 8.53 ; \mathrm{N} 11.85 ; \%$ Found C 66.09; H 8.63; $\mathrm{N}$ 11.95. DSC: $\mathrm{T}_{\mathrm{m}}=70^{\circ} \mathrm{C}$.

Compound 1c: Yield: $88 \% . R M N^{1} H\left(\mathrm{CDCl}_{3}\right) \delta$ (ppm): 0.86 (t, $\left.{ }^{3} \mathrm{~J}=6.4 \mathrm{~Hz}, \underline{\mathrm{CH}}_{3}\left(\mathrm{CH}_{2}\right)_{7} \mathrm{~N}, 3 \mathrm{H}\right)$; $1.26\left(\mathrm{~m}, \mathrm{CH}_{3}\left(\mathrm{C}_{2}\right)_{5} \mathrm{CH}_{2} \mathrm{CH}_{2} \mathrm{~N}, 10 \mathrm{H}\right) ; 1.76(\mathrm{~m}$, $\left.\mathrm{CH}_{3}\left(\mathrm{CH}_{2}\right)_{5} \mathrm{C}_{2} \mathrm{CH}_{2} \mathrm{~N}, 2 \mathrm{H}\right) ; 3.76\left(\mathrm{~s}, \mathrm{OCH}_{3}, 3 \mathrm{H},\right)$; $3.90\left(\mathrm{t},{ }^{3} \mathrm{~J}=7,0 \mathrm{~Hz}, \mathrm{CH}_{3}\left(\mathrm{CH}_{2}\right)_{6} \mathrm{CH}_{2} \mathrm{~N}, 2 \mathrm{H}\right) ; 6.53$ $\left(\mathrm{AB}, J_{A B}=15.85 \mathrm{~Hz}, 1 \mathrm{H}, \mathrm{H}_{\mathrm{a}}\right) ; 7.07\left(\mathrm{~s}, 1 \mathrm{H}, \mathrm{H}_{\mathrm{d}}\right)$; $7.26\left(\mathrm{~s}, 1 \mathrm{H}, \mathrm{H}_{\mathrm{c}}\right) ; 7.54\left(\mathrm{AB}, J_{A B}=15,85 \mathrm{~Hz}, 1 \mathrm{H}\right.$, $\left.\mathrm{H}_{\mathrm{b}}\right)$. MS: DCI $\left(\mathrm{NH}_{3}\right) \mathrm{m} / \mathrm{z}=256, \mathrm{MH}^{+}(100 \%)$. Anal calc (\%) for $\mathrm{C}_{15} \mathrm{H}_{24} \mathrm{~N}_{2} \mathrm{O}_{2}$ : C 68.15; $\mathrm{H}$ 9.15; N 10.60; \% Found C 68.09; H 9.10; N 10.65. DSC: $\mathrm{T}_{\mathrm{m}}=85^{\circ} \mathrm{C}$.

Compound 1d: Yield: $86 \% . R M N^{1} H\left(\mathrm{CDCl}_{3}\right) \delta$ (ppm): 0.87 (t, 3H, CH3); 1.24 (s, 20H, $\left.\mathrm{CH}_{2}\right)$; $1.70\left(\mathrm{~m}, 2 \mathrm{H}, \mathrm{CH}_{2} \beta \mathrm{N}\right) ; 3.76\left(\mathrm{~s}, 3 \mathrm{H}, \mathrm{CH}_{3} \mathrm{O}\right)$; $3.90\left(\mathrm{t}, 2 \mathrm{H}, \mathrm{CH}_{2} \alpha \mathrm{N}\right) ; 6.52(\mathrm{AB}, 1 \mathrm{H}, \mathrm{J}=15 \mathrm{~Hz}$, $\left.\mathrm{H}_{\mathrm{a}}\right) ; 7.07\left(\mathrm{~s}, 1 \mathrm{H}, \mathrm{H}_{\mathrm{d}}\right) ; 7.45\left(\mathrm{~s}, 1 \mathrm{H}, \mathrm{H}_{\mathrm{c}}\right) ; 7.55$ $\left(\mathrm{AB}, 1 \mathrm{H}, \mathrm{J}=15 \mathrm{~Hz}, \mathrm{H}_{\mathrm{b}}\right) \cdot R M N{ }^{13} \mathrm{C}\left(\mathrm{CDCl}_{3}\right) \delta$ (ppm): $168.14\left(\mathrm{C}_{1}\right) ; 138.47$ (C2'); 138.35 (C4'); $136.44\left(\mathrm{C}_{3}\right) ; 121.48\left(\mathrm{C}_{5}\right) ; 115.38\left(\mathrm{C}_{2}\right) ; 51.45$ $\left(\mathrm{CH}_{3} \mathrm{O}\right), 47.37 \quad\left(\mathrm{CH}_{2} \alpha \mathrm{N}\right) ; 31.91 \quad\left(\mathrm{CH}_{2} \beta \mathrm{N}\right)$; 30.95-22.69 $\left(\mathrm{CH}_{2}\right) . \mathrm{MS}\left(\mathrm{DCI}, \mathrm{NH}_{3}\right): \mathrm{m} / \mathrm{z}=321$,

$\mathrm{MH}^{+}(100 \%)$. Anal calc $(\%)$ for $\mathrm{C}_{1} \mathrm{H}_{32} \mathrm{~N}_{2} \mathrm{O}_{2}$, C 71.21 ; H 10.06; N 8.74 ; Found C 71.47 ; H 10.29; N 8,65. DSC: $\mathrm{T}_{\mathrm{m}}=74.5^{\circ} \mathrm{C}$.

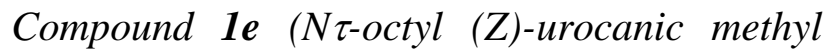
ester)

Compound 1c (150 mg, $10^{-3}$ mol.L $\mathrm{L}^{-1}$ ) was irradiated at $254 \mathrm{~nm}$ in ethanol for $6 \mathrm{~h}$ giving a mixture of the two isomers [70\% (Z) and 30\% $(E)]$. After evaporation of the solvent, the two isomers were separated by flash chromatography (AcOEt / Et $2 \mathrm{O}: 50 / 50 \mathrm{v}: \mathrm{v})$ and $100 \mathrm{mg}\left(3.810^{-4} \mathrm{~mol}\right)$ of $Z$ product was obtained with a yield of $66 \%$.

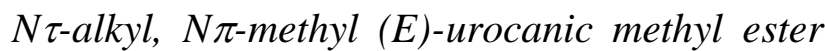
(compounds $2 a, 2 b, 2 c, 2 d, 2 e$ )

General procedure. At low temperature, three equivalents of methyl iodide were added to a solution of compound $\mathbf{1}$ in $50 \mathrm{~mL}$ of dry acetonitrile. The balloon was closed then the mixture was stirred for 7 days at $30^{\circ} \mathrm{C}$. Once the reaction is complete, the excess MeI was taken off under an argon flow and trapped into an aqueous sodium hydroxide solution. The residue, obtained after evaporation of the solvent, was solubilized in a mimimun of $\mathrm{CH}_{2} \mathrm{Cl}_{2}$ and precipitated by adding diethyl ether 
( $\sim 300 \mathrm{~mL})$. Compounds 2 were recovered by filtration.

Compound 2a: Yield: $92 \%$. $R M N^{l} H\left(\mathrm{CDCl}_{3}\right) \delta$ (ppm) : $0.91\left(\mathrm{t},{ }^{3} \mathrm{~J}=7.3 \mathrm{~Hz}, \mathrm{C}_{3} \underline{\mathrm{CH}_{2}} \mathrm{CH}_{2} \mathrm{CH}_{2} \mathrm{~N}\right.$, $3 \mathrm{H}) ; 1.36\left(\mathrm{~m}, \mathrm{CH}_{3} \mathrm{CH}_{2} \mathrm{CH}_{2} \mathrm{CH}_{2} \mathrm{~N}, 2 \mathrm{H}\right) ; 1.90$ (m, $\left.\mathrm{CH}_{3} \mathrm{CH}_{2} \mathrm{CH}_{2} \mathrm{CH}_{2} \mathrm{~N}, 2 \mathrm{H}\right) ; 3,77$ (s, 3H, $\left.\mathrm{OCH}_{3}\right)$; $4.04\left(\mathrm{~s}, 3 \mathrm{H}, \mathrm{N}^{+} \mathrm{CH}_{3}\right) ; 4.34\left(\mathrm{t},{ }^{3} \mathrm{~J}=7.0 \mathrm{~Hz}\right.$, $\left.\mathrm{CH}_{3} \mathrm{CH}_{2} \mathrm{CH}_{2} \underline{\mathrm{CH}}_{2} \mathrm{~N}, 2 \mathrm{H}\right) ; 6.54\left(\mathrm{AB}, J_{A B}=15.85\right.$ $\left.\mathrm{Hz}, 1 \mathrm{H}, \mathrm{H}_{\mathrm{a}}\right) ; 7.26\left(\mathrm{~s}, 1 \mathrm{H}, \mathrm{H}_{\mathrm{d}}\right) ; 7.55\left(\mathrm{AB}, J_{A B}=\right.$ $\left.15.85 \mathrm{~Hz}, 1 \mathrm{H}, \mathrm{H}_{\mathrm{b}}\right) ; 7.58\left(\mathrm{~s}, 1 \mathrm{H}, \mathrm{H}_{\mathrm{c}}\right) . R M N{ }^{13} C$ $\left(\mathrm{CD}_{3} \mathrm{COCD}_{3}, \quad 300 \mathrm{MHz}\right) \quad \delta \quad(\mathrm{ppm}): 13.53$ $\left(\mathrm{CH}_{3}\left(\mathrm{CH}_{2}\right)_{3} \mathrm{~N}\right) ; 19.45\left(\mathrm{CH}_{3} \mathrm{CH}_{2}\left(\mathrm{CH}_{2}\right)_{2} \mathrm{~N}\right) ; 31.94$ $\left(\mathrm{CH}_{3} \mathrm{CH}_{2} \mathrm{CH}_{2} \mathrm{CH}_{2} \mathrm{~N}\right) ; 34.93 \quad\left(\mathrm{~N}^{+} \mathrm{CH}_{3}\right) ; 50.31$ $\left(\mathrm{CH}_{3}\left(\mathrm{CH}_{2}\right)_{2} \mathrm{CH}_{2} \mathrm{~N}\right) ; 52.46\left(\mathrm{CH}_{3} \mathrm{O}\right) ; 122.19\left(\mathrm{C}_{5}\right)$; $124.67\left(\mathrm{C}_{2}\right) ; 125.34\left(\mathrm{C}_{3}\right) ; 130.53\left(\mathrm{C}_{4}\right) ; 137.93$ $\left(\mathrm{C}_{2}\right)$; $165.53\left(\mathrm{C}_{1}\right)$. MS: Electrospray mode + , $\mathrm{m} / \mathrm{z}=223.15(100 \%)$. DSC: $\mathrm{T}_{\mathrm{m}}=146^{\circ} \mathrm{C}$.

Compound $2 \boldsymbol{b}$. Yield: $80 \%$. RMN ${ }^{1} H\left(\mathrm{CDCl}_{3}\right) \delta$ (ppm) : $0.86\left(\mathrm{t},{ }^{3} \mathrm{~J}=6.7 \mathrm{~Hz}, \mathrm{CH}_{3}\left(\mathrm{CH}_{2}\right)_{5} \mathrm{~N}, 3 \mathrm{H}\right)$; $1.31\left(\mathrm{~m}, \mathrm{CH}_{3}\left(\mathrm{CH}_{2}\right)_{3} \mathrm{CH}_{2} \mathrm{CH}_{2} \mathrm{~N}, 6 \mathrm{H}\right) ; 1.96(\mathrm{~m}$, $\left.\mathrm{CH}_{3}\left(\mathrm{CH}_{2}\right)_{4} \mathrm{CH}_{2} \mathrm{CH}_{2} \mathrm{~N}, 2 \mathrm{H}\right) ; 3.78\left(\mathrm{~s}, 3 \mathrm{H}, \mathrm{OCH}_{3}\right)$; $4.09\left(\mathrm{~s}, 3 \mathrm{H}, \mathrm{N}^{+} \mathrm{CH}_{3}\right) ; 4.35\left(\mathrm{t},{ }^{3} \mathrm{~J}=7.0 \mathrm{~Hz}\right.$, $\left.\mathrm{CH}_{3}\left(\mathrm{CH}_{2}\right)_{4} \mathrm{CH}_{2} \mathrm{~N}, 2 \mathrm{H}\right) ; 6.58\left(\mathrm{AB}, J_{A B}=16,10\right.$ $\left.\mathrm{Hz}, 1 \mathrm{H}, \underline{\mathrm{H}}_{\mathrm{A}}\right) ; 7.26\left(\mathrm{~s}, 1 \mathrm{H}, \mathrm{H}_{\mathrm{d}}\right) ; 7.39\left(\mathrm{AB}, J_{A B}=\right.$ $\left.16.10 \mathrm{~Hz}, 1 \mathrm{H}, \mathrm{H}_{\mathrm{b}}\right) ; 7.90\left(\mathrm{~s}, 1 \mathrm{H}, \mathrm{H}_{\mathrm{c}}\right) . \mathrm{MS}$ : Electrospray mode,$+ \mathrm{m} / \mathrm{z}=251.16(100 \%)$. DSC: $\mathrm{T}_{\mathrm{m}}=120^{\circ} \mathrm{C}$

Compound 2c. Yield: $94 \% . R M N{ }^{1} H\left(\mathrm{CDCl}_{3}\right) \delta$ (ppm): 0.85 (t, $\left.{ }^{3} \mathrm{~J}=6,1 \mathrm{~Hz}, \mathrm{CH}_{3}\left(\mathrm{CH}_{2}\right)_{7} \mathrm{~N}, 3 \mathrm{H}\right)$; $1.28\left(\mathrm{~m}, \mathrm{CH}_{3}\left(\mathrm{C}_{2}\right)_{5} \mathrm{CH}_{2} \mathrm{CH}_{2} \mathrm{~N}, 10 \mathrm{H}\right) ; 1.97(\mathrm{~m}$, $\left.\mathrm{CH}_{3}\left(\mathrm{CH}_{2}\right)_{6} \mathrm{C}_{2} \mathrm{CH}_{2} \mathrm{~N}, 2 \mathrm{H}\right) ; 3.82\left(\mathrm{~s}, 3 \mathrm{H}, \mathrm{OCH}_{3}\right)$; $4.09\left(\mathrm{~s}, 3 \mathrm{H}, \mathrm{N}^{+} \mathrm{CH}_{3}\right) ; 4.35\left(\mathrm{t},{ }^{3} \mathrm{~J}=7.3 \mathrm{~Hz}\right.$,
$\left.\mathrm{CH}_{3}\left(\mathrm{CH}_{2}\right)_{6} \mathrm{CH}_{2} \mathrm{~N}, 2 \mathrm{H}\right) ; 6.56\left(\mathrm{AB}, J_{A B}=16.10\right.$ $\left.\mathrm{Hz}, 1 \mathrm{H}, \mathrm{H}_{\mathrm{a}}\right) ; 7.26\left(\mathrm{~s}, 1 \mathrm{H}, \mathrm{H}_{\mathrm{d}}\right) ; 7.36\left(\mathrm{AB}, J_{A B}=\right.$ $\left.16.10 \mathrm{~Hz}, 1 \mathrm{H}, \mathrm{H}_{\mathrm{b}}\right) ; 7.80\left(\mathrm{~s}, 1 \mathrm{H}, \mathrm{H}_{\mathrm{c}}\right) . \mathrm{MS}$ : Electrospray mode,$+ \mathrm{m} / \mathrm{z}=292.28(100 \%)$. DSC: $\mathrm{T}_{\mathrm{m}}=136^{\circ} \mathrm{C}$.

Compound 2d. Yield: $92 \%$. $R M N{ }^{l} H\left(\mathrm{CDCl}_{3}\right) \delta$ (ppm): $0.85\left(\mathrm{t},{ }^{3} \mathrm{~J}=6,1 \mathrm{~Hz}, \mathrm{CH}_{3}\left(\mathrm{CH}_{2}\right)_{11} \mathrm{~N}, 3 \mathrm{H}\right)$; $1.29\left(\mathrm{~m}, \mathrm{CH}_{3}\left(\mathrm{CH}_{2}\right)_{9} \mathrm{CH}_{2} \mathrm{CH}_{2} \mathrm{~N}, 18 \mathrm{H}\right) ; 1.96(\mathrm{~m}$, $\left.\mathrm{CH}_{3}\left(\mathrm{CH}_{2}\right)_{9} \mathrm{C}_{2} \mathrm{CH}_{2} \mathrm{~N}, 2 \mathrm{H}\right) ; 3.80\left(\mathrm{~s}, 3 \mathrm{H}, \mathrm{OCH}_{3}\right)$; $4.09\left(\mathrm{~s}, 3 \mathrm{H}, \mathrm{N}^{+} \mathrm{CH}_{3}\right) ; 4.35\left(\mathrm{t},{ }^{3} \mathrm{~J}=7.3 \mathrm{~Hz}\right.$, $\left.\mathrm{CH}_{3}\left(\mathrm{CH}_{2}\right){ }_{10} \mathrm{CH}_{2} \mathrm{~N}, 2 \mathrm{H}\right) ; 6.57\left(\mathrm{AB}, J_{A B}=16.10\right.$ $\left.\mathrm{Hz}, 1 \mathrm{H}, \mathrm{H}_{\mathrm{a}}\right) ; 7.26\left(\mathrm{~s}, 1 \mathrm{H}, \mathrm{H}_{\mathrm{d}}\right) ; 7.37\left(\mathrm{AB}, J_{A B}=\right.$ $\left.16.10 \mathrm{~Hz}, 1 \mathrm{H}, \mathrm{H}_{\mathrm{b}}\right) ; 7.85\left(\mathrm{~s}, 1 \mathrm{H}, \mathrm{H}_{\mathrm{c}}\right) . \mathrm{MS}$ : Electrospray mode,$+ \mathrm{m} / \mathrm{z}=248.28(100 \%)$.

Compound $2 \boldsymbol{e}$. Yield: $90 \%$. $R M N^{l} H\left(\mathrm{CDCl}_{3}\right) \delta$ (ppm): 0.85 (t, $\left.{ }^{3} \mathrm{~J}=6,1 \mathrm{~Hz}, \mathrm{C}_{3}\left(\mathrm{CH}_{2}\right)_{7} \mathrm{~N}, 3 \mathrm{H}\right)$; $1.28\left(\mathrm{~m}, \mathrm{CH}_{3}\left(\mathrm{CH}_{2}\right)_{5} \mathrm{CH}_{2} \mathrm{CH}_{2} \mathrm{~N}, 10 \mathrm{H}\right) ; 1.97$ (m, $\left.\mathrm{CH}_{3}\left(\mathrm{CH}_{2}\right)_{6} \mathrm{C}_{2} \mathrm{CH}_{2} \mathrm{~N}, 2 \mathrm{H}\right) ; 3.82\left(\mathrm{~s}, 3 \mathrm{H}, \mathrm{OCH}_{3}\right)$; $4.01\left(\mathrm{~s}, 3 \mathrm{H}, \mathrm{N}^{+} \mathrm{CH}_{3}\right) ; 4.35\left(\mathrm{t},{ }^{3} \mathrm{~J}=7.3 \mathrm{~Hz}\right.$, $\left.\mathrm{CH}_{3}\left(\mathrm{CH}_{2}\right)_{6} \mathrm{CH}_{2} \mathrm{~N}, 2 \mathrm{H}\right) ; 6.18\left(\mathrm{AB}, J_{A B}=12.3 \mathrm{~Hz}\right.$, $\left.1 \mathrm{H}, \mathrm{H}_{\mathrm{a}}\right) ; 8.40\left(\mathrm{~s}, 1 \mathrm{H}, \mathrm{H}_{\mathrm{d}}\right) ; 6.75\left(\mathrm{AB}, J_{A B}=12.3\right.$ $\left.\mathrm{Hz}, \quad 1 \mathrm{H}, \mathrm{H}_{\mathrm{b}}\right) ; 10.33 \quad\left(\mathrm{~s}, \quad 1 \mathrm{H}, \mathrm{H}_{\mathrm{c}}\right) . \quad \mathrm{MS}$ : Electrospray mode,$+ \mathrm{m} / \mathrm{z}=292.12(100 \%)$.

Zwitterionic ILs (compounds $\mathbf{3 a}, \mathbf{3 b}, \mathbf{3 c}, \mathbf{3 d}, \mathbf{3 e}$ ) General procedure. To an aqueous solution of compound 2 was added Amberlite IRA 400 resin regenerated before use $(930 \mathrm{~g}$ of resin per mole of compound). The reaction is carried out at ambient temperature for $2 \mathrm{~h}$. The product was obtained after lyophilization and drying under vacuum over $\mathrm{P}_{2} \mathrm{O}_{5}$. 
Compound 3a. Yield: $95 \%$. $R M N{ }^{1} H\left(\mathrm{CD}_{3} \mathrm{OD}\right.$, $250 \mathrm{MHz}) \delta(\mathrm{ppm}): 1.00\left(\mathrm{t},{ }^{3} \mathrm{~J}=7.3 \mathrm{~Hz}\right.$, $\left.\mathrm{C}_{3} \mathrm{CH}_{2} \mathrm{CH}_{2} \mathrm{CH}_{2} \mathrm{~N}, \quad 3 \mathrm{H}\right) ; \quad 1.39 \quad(\mathrm{~m}$, $\left.\mathrm{CH}_{3} \mathrm{CH}_{2} \mathrm{CH}_{2} \mathrm{CH}_{2} \mathrm{~N}, \quad 2 \mathrm{H}\right) ; \quad 1.87 \quad$ (m, $\left.\mathrm{CH}_{3} \mathrm{CH}_{2} \mathrm{CH}_{2} \mathrm{CH}_{2} \mathrm{~N}, 2 \mathrm{H}\right) ; 3.90$ (s, 3H, N $\left.{ }^{+} \mathrm{CH}_{3}\right)$; $4.19\left(\mathrm{t},{ }^{3} \mathrm{~J}=7.4 \mathrm{~Hz}, \mathrm{CH}_{3} \mathrm{CH}_{2} \mathrm{CH}_{2} \underline{\mathrm{CH}}_{2} \mathrm{~N}, 2 \mathrm{H}\right)$; $6.57\left(\mathrm{AB}, J_{A B}=15.90 \mathrm{~Hz}, 1 \mathrm{H}, \mathrm{H}_{\mathrm{a}}\right) ; 7.20(\mathrm{AB}$, $\left.J_{A B}=15.90 \mathrm{~Hz}, 1 \mathrm{H}, \mathrm{H}_{\mathrm{b}}\right) ; 7.99\left(\mathrm{~s}, 1 \mathrm{H}, \mathrm{H}_{\mathrm{c}}\right) . R M N$ ${ }^{13} \mathrm{C} \quad\left(\mathrm{CD}_{3} \mathrm{COCD}_{3}\right) \quad \delta \quad$ (ppm): 12.41 $\left(\mathrm{CH}_{3}\left(\mathrm{CH}_{2}\right)_{3} \mathrm{~N}\right) ; 19.08\left(\mathrm{CH}_{3} \underline{C H}_{2}\left(\mathrm{CH}_{2}\right)_{2} \mathrm{~N}\right) ; 31.54$ $\left(\mathrm{CH}_{3} \mathrm{CH}_{2} \mathrm{CH}_{2} \mathrm{CH}_{2} \mathrm{~N}\right) ; 33.11 \quad\left(\mathrm{~N}^{+} \mathrm{CH} 3\right) ; 49.38$ $\left(\mathrm{CH}_{3} \mathrm{CH}_{2} \mathrm{CH}_{2} \mathrm{CH}_{2} \mathrm{~N}\right) ; 119.56\left(\mathrm{C}_{5},\right) ; 120.51\left(\mathrm{C}_{2}\right)$; $132.60\left(\mathrm{C}_{3}\right) ; 132.70\left(\mathrm{C}_{2}\right) ; 171.52\left(\mathrm{C}_{1}\right)$. Anal calc (\%) for $\mathrm{C}_{11} \mathrm{H}_{16} \mathrm{~N}_{2} \mathrm{O}_{2}$ : C 63.44; $\mathrm{H} 7.74 ; \mathrm{N} 13.45$; $\%$ Found C 63.05; H 8.11; N 13.23. DSC: $\mathrm{T}_{\mathrm{m}}=81.5^{\circ} \mathrm{C}$.

Compound 3b. Yield: 97 \%. RMN ${ }^{1} \mathrm{H}\left(\mathrm{CD}_{3} \mathrm{OD}\right)$ $\delta(\mathrm{ppm}): 0.92\left(\mathrm{t},{ }^{3} \mathrm{~J}=6.8 \mathrm{~Hz}, \mathrm{C}_{3}\left(\mathrm{CH}_{2}\right)_{5} \mathrm{~N}, 3 \mathrm{H}\right)$; $1.37\left(\mathrm{~m}, \mathrm{CH}_{3}\left(\mathrm{CH}_{2}\right)_{3} \mathrm{CH}_{2} \mathrm{CH}_{2} \mathrm{~N}, 6 \mathrm{H}\right) ; 1.90$ (m, $\left.\mathrm{CH}_{3}\left(\mathrm{CH}_{2}\right)_{3} \mathrm{CH}_{2} \mathrm{CH}_{2} \mathrm{~N}, \quad 2 \mathrm{H}\right) ; \quad 3.91 \quad(\mathrm{~s}, \quad 3 \mathrm{H}$, $\left.\mathrm{N}^{+} \mathrm{CH}_{3}\right) ; 4.20\left(\mathrm{t},{ }^{3} \mathrm{~J}=7.4 \mathrm{~Hz}, \mathrm{CH}_{3}\left(\mathrm{CH}_{2}\right)_{4} \underline{\mathrm{C}}_{2} \mathrm{~N}\right.$, $2 \mathrm{H}) ; 6.55\left(\mathrm{AB}, J_{A B}=16.00 \mathrm{~Hz}, 1 \mathrm{H}, \mathrm{H}_{\mathrm{a}}\right) ; 7.19$ $\left(\mathrm{AB}, J_{A B}=16.00 \mathrm{~Hz}, 1 \mathrm{H}, \mathrm{H}_{\mathrm{b}}\right) ; 8.00\left(\mathrm{~s}, 1 \mathrm{H}, \mathrm{H}_{\mathrm{c}}\right)$. $\begin{array}{llllll}R M N & { }^{13} \mathrm{C} & \left(\mathrm{CD}_{3} \mathrm{COCD}_{3}\right) & \delta & \text { (ppm): } & 12.48\end{array}$ $\left(\mathrm{CH}_{3}\left(\mathrm{CH}_{2}\right)_{5} \mathrm{~N}\right) ; 19.48\left(\mathrm{CH}_{3} \underline{\mathrm{CH}}_{2}\left(\mathrm{CH}_{2}\right)_{3} \mathrm{~N}\right) ; 31.64$ $\left(\mathrm{CH}_{3} \mathrm{CH}_{2} \mathrm{CH}_{2} \mathrm{CH}_{2} \mathrm{~N}\right) ; 33.21 \quad\left(\mathrm{~N}^{+} \mathrm{CH} 3\right) ; 50.23$ $\left(\underline{\mathrm{CH}}_{2} \mathrm{~N}\right) ; 120.58\left(\mathrm{C}_{5},\right) ; 123.51\left(\mathrm{C}_{2}\right) ; 128.60\left(\mathrm{C}_{3}\right)$; $135.70\left(\mathrm{C}_{2}\right) ; 168.52\left(\mathrm{C}_{1}\right)$. Anal calc $(\%)$ for $\mathrm{C}_{13} \mathrm{H}_{20} \mathrm{~N}_{2} \mathrm{O}_{2}$ : C 66.07; $\mathrm{H}$ 8.53; N 11.85; \% Found C 65.65; H 8.99; N 11.73. DSC: $\mathrm{T}_{\mathrm{m}}=75$ ${ }^{\circ} \mathrm{C}$.
Compound 3c. Yield: $96 \%$. RMN ${ }^{1} \mathrm{H}\left(\mathrm{CD}_{3} \mathrm{OD}\right)$ $\delta(\mathrm{ppm}): 1.17\left(\mathrm{t},{ }^{3} \mathrm{~J}=7.1 \mathrm{~Hz}, \mathrm{CH}_{3}\left(\mathrm{CH}_{2}\right)_{7} \mathrm{~N}, 3 \mathrm{H}\right)$; $1.37\left(\mathrm{~m}, \mathrm{CH}_{3}\left(\mathrm{C}_{2}\right)_{5} \mathrm{CH}_{2} \mathrm{CH}_{2} \mathrm{~N}, 10 \mathrm{H}\right) ; 1.90(\mathrm{~m}$, $\left.\mathrm{CH}_{3}\left(\mathrm{CH}_{2}\right)_{5} \mathrm{C}_{2} \mathrm{CH}_{2} \mathrm{~N}, \quad 2 \mathrm{H}\right) ; \quad 3.91 \quad(\mathrm{~s}, \quad 3 \mathrm{H}$, $\left.\mathrm{N}^{+} \mathrm{CH}_{3}\right) ; 4.19\left(\mathrm{t},{ }^{3} \mathrm{~J}=7.4 \mathrm{~Hz}, \mathrm{CH}_{3}\left(\mathrm{CH}_{2}\right)_{6} \mathrm{C}_{2} \mathrm{~N}\right.$, $2 \mathrm{H}) ; 6.57\left(\mathrm{AB}, J_{A B}=16.0 \mathrm{~Hz}, 1 \mathrm{H}, \mathrm{Ha}\right) ; 7.19$ $\left(\mathrm{AB}, J_{A B}=16.0 \mathrm{~Hz}, 1 \mathrm{H}, \mathrm{H}_{\mathrm{b}}\right) ; 8.00\left(\mathrm{~s}, 1 \mathrm{H}, \mathrm{H}_{\mathrm{c}}\right)$. Anal calc (\%) for $\mathrm{C}_{15} \mathrm{H}_{24} \mathrm{~N}_{2} \mathrm{O}_{2}$ : C 68.15; $\mathrm{H} 9.15$; N 10.60; \% Found C 67.35; H 9.33; N 10.23. DSC: $\mathrm{T}_{\mathrm{m}}=41^{\circ} \mathrm{C}$.

Compound 3d. Yield: 91 \%. $R M N{ }^{1} H\left(\mathrm{CD}_{3} \mathrm{OD}\right)$ $\delta$ (ppm): $0.89\left(\mathrm{t},{ }^{3} \mathrm{~J}=7,1 \mathrm{~Hz}, \mathrm{C}_{3}\left(\mathrm{CH}_{2}\right)_{11} \mathrm{~N}\right.$, $3 \mathrm{H}) ; 1.36\left(\mathrm{~m}, \mathrm{CH}_{3}\left(\mathrm{CH}_{2}\right)_{9} \mathrm{CH}_{2} \mathrm{CH}_{2} \mathrm{~N}, 10 \mathrm{H}\right) ; 1.92$ (m, $\left.\mathrm{CH}_{3}\left(\mathrm{CH}_{2}\right)_{9} \mathrm{CH}_{2} \mathrm{CH}_{2} \mathrm{~N}, 2 \mathrm{H}\right) ; 3.90(\mathrm{~s}, 3 \mathrm{H}$, $\left.\mathrm{N}^{+} \mathrm{CH}_{3}\right) ; 4.18\left(\mathrm{t},{ }^{3} \mathrm{~J}=7.4 \mathrm{~Hz}, \mathrm{CH}_{3}\left(\mathrm{CH}_{2}\right)_{6} \mathrm{C}_{2} \mathrm{~N}\right.$, $2 \mathrm{H}) ; 6.58\left(\mathrm{AB}, J_{A B}=16,0 \mathrm{~Hz}, 1 \mathrm{H}, \mathrm{H}_{\mathrm{a}}\right) ; 7.19$ $\left(\mathrm{AB}, J_{A B}=16.0 \mathrm{~Hz}, 1 \mathrm{H}, \mathrm{H}_{\mathrm{b}}\right) ; 7,99\left(\mathrm{~s}, 1 \mathrm{H}, \mathrm{H}_{\mathrm{c}}\right)$. Anal calc (\%) for $\mathrm{C}_{19} \mathrm{H}_{32} \mathrm{~N}_{2} \mathrm{O}_{2}: \mathrm{C} 71.21 ; \mathrm{H}$ 10.06; N 8.74; \% Found C 70.72; H 10.43; N 8.73. DSC: $\mathrm{Tm}=41^{\circ} \mathrm{C} \cdot$ DSC: $\mathrm{T}_{\mathrm{m}}=69^{\circ} \mathrm{C}$

Compound 3e. Yield: 97\%. $R M N^{1} H\left(\mathrm{CDCl}_{3}\right) \delta$ (ppm): $0.81\left(\mathrm{t},{ }^{3} \mathrm{~J}=6.1 \mathrm{~Hz}, \underline{\mathrm{H}}_{3}\left(\mathrm{CH}_{2}\right)_{7} \mathrm{~N}, 3 \mathrm{H}\right)$; $1.24\left(\mathrm{~m}, \mathrm{CH}_{3}\left(\mathrm{CH}_{2}\right)_{5} \mathrm{CH}_{2} \mathrm{CH}_{2} \mathrm{~N}, 10 \mathrm{H}\right) ; 1.74(\mathrm{~m}$, $\left.\mathrm{CH}_{3}\left(\mathrm{CH}_{2}\right)_{6} \mathrm{C}_{2} \mathrm{CH}_{2} \mathrm{~N}, 2 \mathrm{H}\right) ; 3.70\left(\mathrm{~s}, 3 \mathrm{H}, \mathrm{OCH}_{3}\right)$; $3.86\left(\mathrm{~s}, 3 \mathrm{H}, \mathrm{N}^{+} \mathrm{CH}_{3}\right) ; 4.35\left(\mathrm{t},{ }^{3} \mathrm{~J}=7.3 \mathrm{~Hz}\right.$, $\left.\mathrm{CH}_{3} \mathrm{CH}_{2} \mathrm{CH}_{2} \mathrm{CH}_{2} \mathrm{~N}, 2 \mathrm{H}\right) ; 5.72\left(\mathrm{AB}, J_{A B}=16.10\right.$ $\mathrm{Hz}, 1 \mathrm{H}, \mathrm{Ha}) ; 6.95\left(\mathrm{AB}, J_{A B}=16.10 \mathrm{~Hz}, 1 \mathrm{H}\right.$, $\left.\mathrm{H}_{\mathrm{b}}\right) ; 7.40\left(\mathrm{~s}, 1 \mathrm{H}, \mathrm{H}_{\mathrm{d}}\right) ; 8.36\left(\mathrm{~s}, 1 \mathrm{H}, \mathrm{H}_{\mathrm{c}}\right)$. Anal calc (\%) for $\mathrm{C}_{15} \mathrm{H}_{24} \mathrm{~N}_{2} \mathrm{O}_{2}, \mathrm{H}_{2} \mathrm{O}$ : C 63.80; $\mathrm{H} 9.28 ; \mathrm{N}$ 9.92; \% Found C 63.95; H 9.53; N 10.03. DSC: $\mathrm{T}_{\mathrm{m}}=-20^{\circ} \mathrm{C}$. 


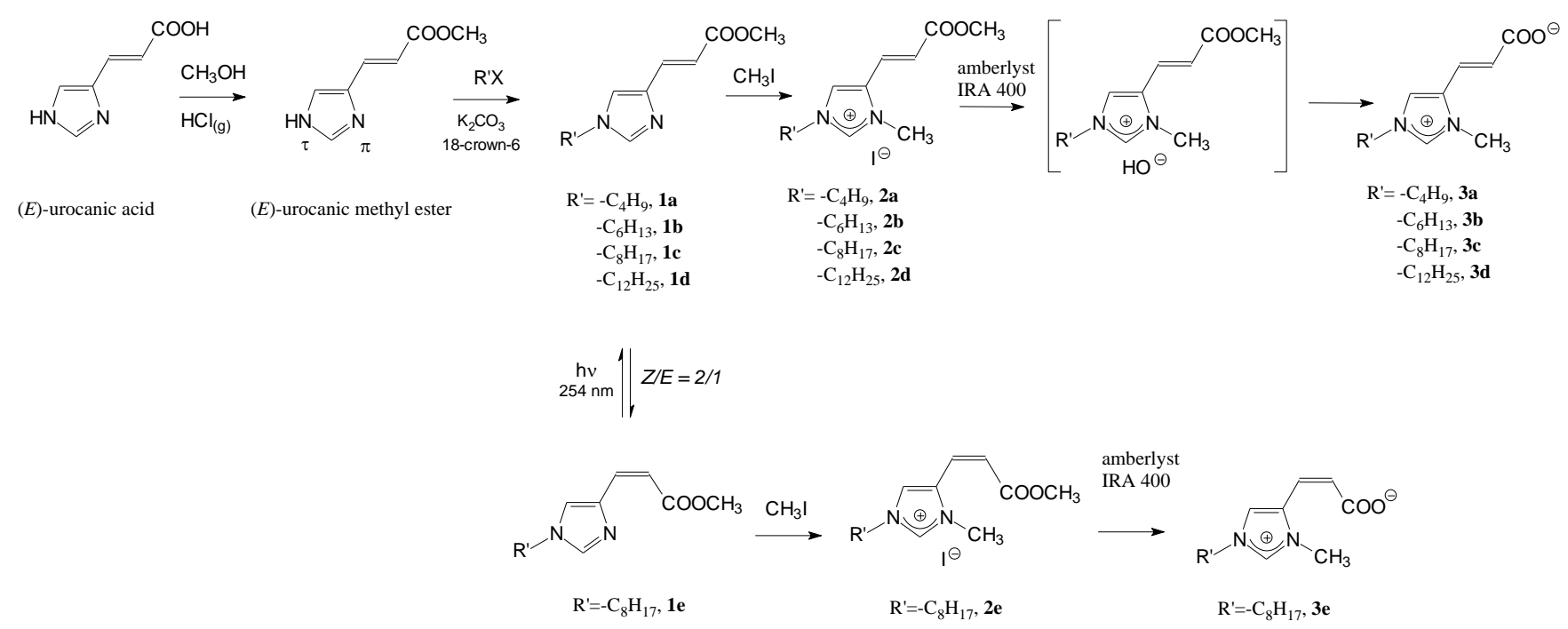

Scheme 2. Synthetic route for the preparation of ZILs based on urocanic moiety E or Z.

\section{Results and discussion}

The synthetic route used to obtain ZILs was described in Scheme 2. (E)-urocanic acid is insoluble in organic solvents, so the carboxylic function was first esterified to give $(E)$-urocanic methyl ester. As (E)-urocanic acid exists in two tautomeric forms, $\mathrm{N}$-alkylation can occur at two sites, le $\mathrm{N}(\tau)$ and $\mathrm{N}(\pi)$ nitrogen atoms. This question is related to the general problem concerning the $\mathrm{N}$-alkylation of imidazole derivatives substituted in position 4(5). In our previous studies, we have optimized conditions which allowed to obtain the $\mathrm{N}(\tau)$-alkylation of the methyl urocanate, fastly, regiospecifically, with quantitative yields.[19] Hence, under solidliquid phase transfer conditions, various bromoalkanes (n-bromobutane, n-bromohexane, n-bromooctane and n-bromododecane) were allowed to react with $(E)$-methyl urocanate in the presence of potassium carbonate, with crown ether acting as catalyst. By this regioselective $\mathrm{N} \tau$-alkylation, compounds 1a-1d were obtained in excellent yields $(\sim 90 \%)$.

In the following step, the action of iodomethane on compounds 1a-1d led to the $N \tau, N \pi$ disubstituted products (compounds 2a-2d) with yields between 80 and $94 \%$. Then the iodide counter-ion was exchanged by a hydroxide anion thanks to a basic amberlite resin (IRA 400). The basic hydrolysis occurred spontaneously leading to (E)-1-alkyl-3-methyl4-propen-carboxylate imidazoliums with a butyl (3a), hexyl (3b), octyl (3c) or dodecyl (3d) chain in quantitative yields. Overall yields are $60,53,60,55 \%$ respectively.

The melting points of the compounds 3a-3d were measured by DSC. The values obtained are reported in Table 2. 
Table 2. Values of melting point of ZILs synthesized determined by DSC

\begin{tabular}{|c|c||c|c|}
\hline compounds & $\begin{array}{c}\mathrm{T}_{\mathrm{m}} /{ }^{\circ} \mathrm{C}^{*} \\
\text { E isomers }\end{array}$ & compound & $\begin{array}{c}\mathrm{T}_{\mathrm{m}} /{ }^{\circ} \mathrm{C}^{*} \\
\mathrm{Z} \text { isomer }\end{array}$ \\
\hline 3a & 81.5 & & \\
\hline 3b & 75.0 & & \\
\hline 3c & 41.0 & $\mathbf{3 e}$ & -20 \\
\hline 3d & 69.0 & & \\
\hline \multicolumn{2}{|c|}{ * onset temperature obtained from extrapolation of measured } \\
\hline
\end{tabular}

values at a $0^{\circ} \mathrm{C} / \mathrm{min}$ heating rate.

Comparatively to the ZILs previously synthesized in literature (see Figure 1 and 2), the melting points obtained were found at moderate temperatures and in all cases below $100^{\circ} \mathrm{C}$. In addition, these temperatures were strongly dependent on the alkyl chain length. As shown on figure 3 , a minimum value equal to $41^{\circ} \mathrm{C}$ for $\mathrm{R}^{\prime}=\mathrm{C}_{8} \mathrm{H}_{17}$ (3c) was obtained. This effect is ascribed to structural effect, indeed, the size and the symmetry of ions directly impacts upon melting point values. This trend was previously described for 1-alkyl-3-methyl imidazolium salts [2] for which a minimum melting temperature was obtained for $\mathrm{N}$-hexyl and $\mathrm{N}$-octyl chains.

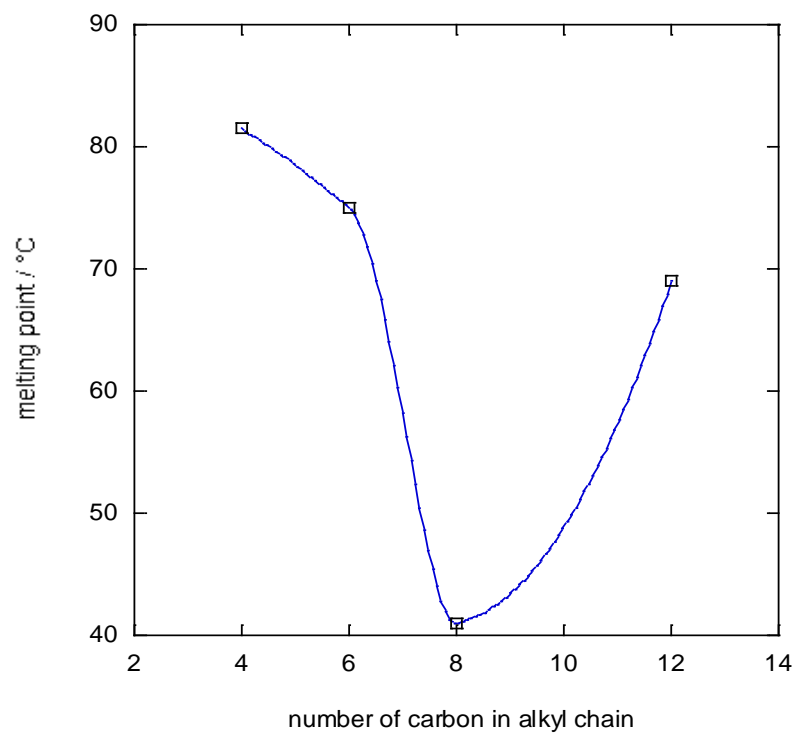

Figure 3. Melting points for (E)-3-(1-alkyl-3-methyl-1Himidazol-4-ium)-2-methylpropenoate zwitterions (3a-3d) as a function of alkyl chain length substituted on the $\mathrm{N}(\tau)$ position.

To further decrease the melting point of these ZILs, we decided to perform photoisomerization of $\mathrm{E}$ isomers to form the $\mathrm{Z}$. The direct photoisomerization of $\mathrm{E}$ isomers (3a3d) did not lead to the formation of corresponding $\mathrm{Z}$ isomers. Therefore, we decided to perform the synthesis of (Z)-1-octyl-3methylurocanate (3e) from compound 1c. Under irradiation at $254 \mathrm{~nm}$ in ethanol, 1c undergoes photoisomerization to give a mixture of the $\mathrm{E}$ and $\mathrm{Z}$ corresponding isomers (1c and $\mathbf{1 e}$ ), which were separated on a silica column (at photostationary equilibrium $E / Z=1: 2)$. After separation, 1e was obtained with a yield of $66 \%$. The two following steps, quaternization and iodide exchange, gave the (Z)-3-(1-octyl-3methyl-1H-imidazol-4-ium)-2methylpropenoate (3e) which is liquid as room 
temperature. The overall yield of $\mathbf{3 e}$ is $38 \%$. The melting point of $\mathbf{3 e}$ determined by DSC is of $20^{\circ} \mathrm{C}$.

\section{Conclusions}

A route to zwitterionic ionic liquids bearing carboxylate function using a natural product as starting material $(E)-3-(1 \mathrm{H}-$ imidazol4-yl)-2-propionic acid or (E)-urocanic acid has been established. For all these compounds, melting points below $100^{\circ} \mathrm{C}$ were observed. The lowest melting point among the $E$ isomer $\left(41^{\circ} \mathrm{C}\right)$ is sufficiently low to enable applications as a solvent. This temperature can be further decreased by using $Z$ isomer down to $-20^{\circ} \mathrm{C}$. This opens promising opportunities to use such ZILs as reaction medium.

\section{References}

[1] Angell C, Ansari Y, Zhao Z. Ionic liquids:past, present and future. Faraday Discuss 2012; $154: 9-27$

[2] Wasserscheid P, Welton T. Ionic liquids in synthesis. Weinheim: Wiley-VCH, 2008.

[3] Kokorin A. Ionic Liquids: Applications and Perspectives. InTech; 2011.

[4] Quartarone E, Mustarelli P. Electrolytes for solid-state lithium rechargeable batteries: recent advances and perspectives. Chem. Soc. Rev. 2011;40:2525-2540

[5] Devanathan R. Recent developments in proton exchange membranes for fuel cells Energy Environ.Sci. 2008;1:101-119

[6] Yoshizawa M, Hirao M, Ito-Akita K, Ohno H. Ion conduction in zwitterionic-type molten salts and their polymers. J. Mater. Chem. 2001; 11:1057-1062
[7] Yoshizawa-Fujita M, Tamura T, Takeoka Y, Rikukawa M. Low-melting zwitterion: effect of oxyethylene units on thermal properties and conductivity. Chem Commun. 2011;47:2345-2347

[8] Yoshizawa M, Ohno H. Anhydrous proton transport system based on zwitterionic liquid and HTFSI. Chem. Comm. 2004:1828-1829.

[9] Nguyen DQ, Hwang J, Lee JS, Kim H, Lee H, Cheong M, Lee B, Sik Kim H. Multi-functional zwitterionic compounds as additives for lithium battery electrolytes. Electrochem. Commun. 2007;9:109-114.

[10] Fei ZF, Zhao DB, Geldbach TJ, Scopelliti R, Dyson PJ. Brønsted Acidic Ionic Liquids and Their Zwitterions: Synthesis, Characterization and $\mathrm{p} K_{\mathrm{a}}$ Determination. Chem. Eur. J. 2004;10:4886-4893

[11] Lissner E, de Souza WF, Ferrera B., Dupont J. Oxidative Desulfurization of Fuels with Task-Specific Ionic Liquids. ChemSusChem 2009; 2:962-964

[12] Nockemann P, Thijs B, Pittois S, Thoen J, Glorieux C, Van Hecke K., Van Meervelt L, Kirchner B, Binnemans K.. Task-Specific Ionic Liquid for Solubilizing Metal Oxides J. Phys. Chem. B 2006; 110: $20978-20992$

[13] Lin JCY, Huang CJ, Lee YT, Lee KM, Lin IJB. Carboxylic acid functionalized imidazolium salts: sequential formation of ionic, zwitterionic, acidzwitterionic and lithium salt-zwitterionic liquid crystals. J. Mater. Chem. 2011; 21:8110-8121

[14] Fei Z, Zhao D, Geldbach TJ, Scopelliti R, Dyson PJ. Bronted acidic ionic liquids and their zwitterions: synthesis, characterization and $\mathrm{pK}_{\mathrm{a}}$ determination. Chem. Eur. J. 2004;10:4886-4893

[15] Holbrey JD, Reichert WM, Tkatchenko I, Bouajila E, Walter O, Tommasi I, Rogers RD. 1,3Dimethylimidazolium-2-carboxylate: the unexpected synthesis of an ionic liquid precursor and carbene-CO2 adduct. Chem. Comm. 2003;28-29.

[16] Mehler A, Tabor H. Isolation of N-formyl$L$-glutamic acid as an intermediate in the enzymatic 
degradation of $L$-histidine. J Biol Chem. 1953;201:775-

785

[17] Tabachnick J. Urocanic acid, the major acid-soluble, ultraviolet-absorbing compound in guinea pig epidermis. Arch. Biochem. Biophys. 1957;70:295-298

[18] Anglin JH. Urocanic acid, a natural sunscreen. Cosmet and toiletries 1976;91:47-4.

[19] Lauth-de Viguerie N, Seegueeva N, Damiot M, Mawlawi H, Riviere M, Lattes A. Selective Nalkylation of (E)-urocanic acid. Heterocycles 1994;37:1561-1573 